\title{
A MICROFLUIDICS-BASED INSTRUMENT FOR CYTOMECHANICAL STUDIES OF BLOOD
}

\author{
M. Tracey (1), N. Sutton (1), I. Johnston (1) and W. Doetzel (2) \\ (1) Science and Technology Research Centre - University of Hertfordshire - College Lane - Hatfield - Hertfordshire - \\ AL109AB, UK - Email m.tracey@herts.ac.uk \\ (2) Fakultat fur Elektrotechnik und Informationstechnik - Technische Universitat Chemnitz - Reichenhainer Strasse 70 - \\ 09126 Chemnitz, Germany - Email Wolfram.Doetzel@infotech.tu-chemnitz.de
}

\begin{abstract}
A complete instrument for the measurement of erythrocyte (red blood cell) flow in microchannels is presented. The instrument measures circa 1500 cells on a cell-by-cell basis in an array of microchannels. Microchannel dimensions and operating pressures are physiologically-analogous. Resulting bì-variate data, obtained by real-time image processing, describes cell flow with a unique volume-velocity space representation which provides detailed insights into both cell flow resistance (independent of cell volume) and the presence of pathological sub-populations.

We commence by discussing the instrument's design, with particular attention to the microfluidics. We proceed to report analyses of the instrument's reproducibility, the characterisation of normal blood, and our ability to detect artificial sub-populations of cells with rigid membranes produced by chemically modifying a fraction of the analyte. Having validated the performance of the instrument, we then present a clinical result from a transfused thalassaemic displaying highly irregular microrheological metrics.
\end{abstract}

\section{INTRODUCTION}

\section{A. Microvascular Haemodynamics}

Blood is a complex two phase fluid consisting of solid bodies, the blood cells, in suspension within an aqueous liquid phase, the plasma. To perform its primary physiological roles of nutrient transport, waste transport and deployment of white cells, the blood must flow through the vast, diverse network of vessels which constitute the circulatory system. At one extreme, human blood vessels are several centimetres in diameter and blood flows through this region as a continuous body obeying bulk fluid laws. This situation ceases to apply within the capillary beds, where the exchange of nutrient gases is performed. Here vessel diameters are just a few microns: smaller than the blood cells themselves. Within this region, cells must deform to squeeze through the vessels in single-file to perform their critical roles.

By far the most numerous of blood cells is the erythrocyte. These consist of an under-filled, viscoelastic membrane encasing a viscous liquid interior. At rest these highly specialised cells take the form of a biconcave disk, typically of $8 \mu \mathrm{m}$ diameter and $2 \mu \mathrm{m}$ thickness.

The ability of the erythrocyte to deform is dependent on the intrinsic mechanical properties of its constituent parts and extrinsic factors characterised by its volume and specific morphology. Within the vascular network, blood cells are subjected to static, time invariant deformation but they must also deform dynamically: in response to timevarying deformational forces. This is a critical mechanism by which erythrocytes can transit the tortuous environment of the capillary beds.

Microhaemorheology is the study of the mechanisms of cellular deformation and blood flow at the single cell level. Much experimental work has concentrated on measuring the intrinsic mechanical properties of erythrocytes [1] and the ability of the blood cells to pass through (non microengineered) filter-type structures [2].

Measurements of cellular mechanical properties of several pathological states have indicated impaired cellular deformation characteristics [1]. Ultimately however, a cell's ability to transit a microvessel environment is perhaps a more telling indication of the physiological implications these cytomechanical changes may have. Unfortunately little work is reported on in-vitro measurements of blood cells flowing under controlled, physiologically-analogous conditions. Here we present a microhaemeorheological instrument designed to approach these issues.

\section{B. Cyto-Manipulation on Chip}

Whilst the potential of whole cell handling with microfluid systems is recognised, a limited volume of work has been reported thus far. This work has centred upon three areas: cell mechanics; DNA extraction; and cell sorting and handling.

Cell mechanical studies probably represented the earliest of these areas to be investigated. Original work employed $\mathrm{KOH}$-etched silicon as a discrete filter component without cell-by-cell data extraction [3]. Subsequently we integrated silicon flow structures into an instrument employing image processing to provide cell-bycell data extraction [4], [5], [6]. Two groups [7] and [8] reported the studies of flow in microfabricated vessels of larger than microvascular dimensions. In [9] a device employing repeated banks of microvascular-dimensioned channels was employed to investigate an hypothesis that erythrocyte membrane deformability changed in response to repeated deformation.

The desirability of being able to conduct DNA analyses based upon low trauma cell sampling via finger-prick sampling or buccal swabs, with their concomitant low sample volumes, has lead several groups to investigate

$621^{\text {st }}$ Annual International IEEE-EMBS Special Topic Conference on Microtechnologies in Medicine \& Biology October 12-14, 2000, Lyon, France - 0-7803-6603-4/00/\$10.00@2000 IEEE 
microfluid-based white cell separation and subsequent onchip lysis for DNA extraction. One approach has been to employ filter structures to pass erythrocytes whilst trapping white cells [10].

Amongst examples of cell sorting and handling, [11] reports the separation of rare foetal blood cells that have migrated into the maternal circulation thereby promising non-invasive foetal screening. Reference [12] reports the use of microfluidics for embryo manipulation in, for instance, IVF and cloning.

We have continued to develop our blood cell analysis system to the point where it can reliably assess samples and function as a haemorheological tool. In the following section we discuss the evolution of the instrument to this point.

\section{THE INSTRUMENT}

The instrument, shown in Fig. 1 can be delineated into four distinct topics: microfluidics, macrofluidics, real-time image processing, and data post-processing.

\section{A. Microfluidic Considerations}

Microfluidics, particularly in the guise of "lab-on-achip' is highly topical. Typical devices (for instance electrophoresis chips) are often characterised by relatively large feature sizes, the use of glass and plastics as substrate materials, and the use of electrokinetics as a fluid transport mechanism. Work reported to date is predominately liquid phase with more recent developments focusing upon a suspended phase in the form of bead supports for solid phase chemistry [13].

Our devices differ somewhat. They require fine feature sizes to provide the precise control of the cell's environment. They employ hydrostatic pressure-driven fluid transport to simulate the environment cells encounter invivo. Whilst the choice of silicon-glass-silicon technology is an evolution of earlier designs it is still the only nonresearch technology offering the ability to implement two-level fluidics, sub-micron precision and anisotropic structuring techniques.

Our measurements concern a delicate suspended phase of blood cells that require care with fluid flow conditions in order to avoid cell lysis and to avoid biasing of sample statistics. These restrictions are less onerous in single phase liquid systems.

\section{B. Microfluidic Device Fabrication and Design}

The particular devices reported here, fabricated at the $Z \mathrm{fM}$, Technische Universitat Chemnitz, employ doublesided silicon wafer processing with both faces of the wafer being sealed by anodic-bonded glass: the upper surface providing a viewing window, the lower having ultrasonically-drilled feed vias formed in the glass prior to bonding, thereby providing fluid connectivity. Device design considerations are further detailed in [14] and [15].

Fig. 2 shows the upper face of the silicon featured with a

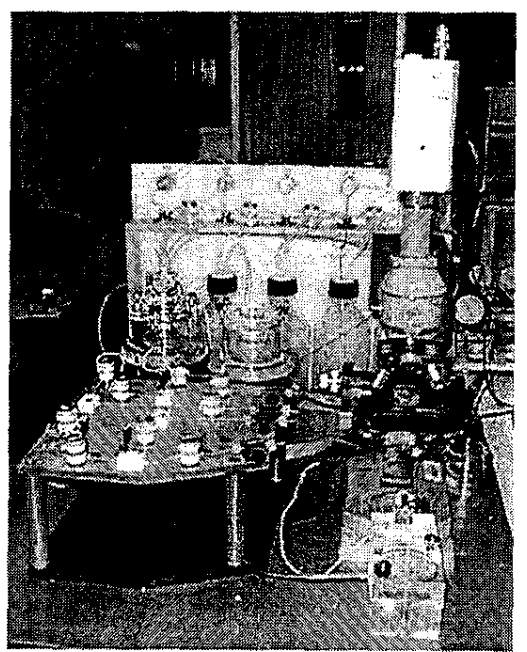

Fig. 1. The instrument, detailing the macrofluidic circuit and the hydrostatic head mechanism to the left. The microfluidic device is positioned on the microscope stage.

four-sided array of approximately 1500, $100 \mu \mathrm{m}$ long, circa $3.5 \mu \mathrm{m}$ section (depending upon channel design and device variant) plasma etched, flow channels. The array, detailed in Fig. 3, is fed by a central reservoir area of circa $17 \mu \mathrm{m}$ depth formed by anisotropic wet etching.

In the centre of the reservoir is a feed-via to the opposite wafer face, in fact this intercepts a similar via emerging from that face in order to minimise cross-sectional area and hence maximise suspension flow velocity and minimise cell sedimentation.

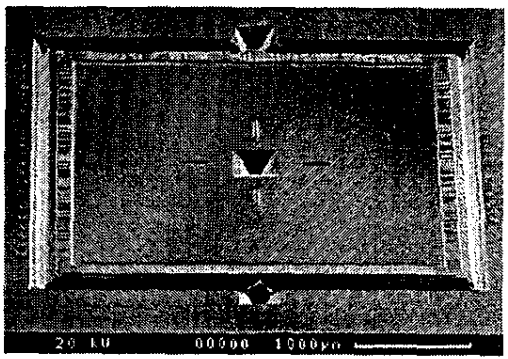

Fig. 2. SEM of a flow device prior to anodic bonding of the glass cover. The device inlet port is in the centre of the picture. The two outlet ports are at the top and bottom of the picture.

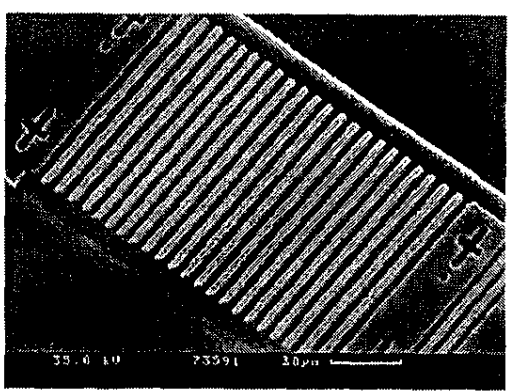

Fig. 3. SEM of an array of silicon microengineered flow channels. The wet-etch forming the vias operates on both wafer 
faces concurrently and also, via crystal-plane self-limiting, forms flow-bypass manifolding on the lower face (this is a critical aspect of the micro-macro interfacing and is discussed below) as well as a waste collection 'gutter' outside the square array on the upper face. The completed silicon wafers are thermally oxidised to present a well defined hydrophilic surface and then anodic bonded and diced.

Fig. 4 shows the resulting device mounted on a small printed circuit board (PCB) containing a closed-loop temperature control system to hold the chip at $37 \mathrm{C}$ (or any other desired temperature). Device heating is via a goldplated copper mounting pad with chip resistors serving as heating elements. Fluid connectivity is provided by seven $0.75 \mathrm{~mm}$ o.d., $0.25 \mathrm{~mm}$ i.d. nylon tubes fitted into ultrasonically drilled holes and sealed by epoxy adhesive.

\section{Macrofluid System Design}

Instrument miniaturisation was not our objective: rather we sought to benefit from the precision of the microfluidic flow device. Accordingly the external fluid system consists of miniature, but conventional, components.

When dealing with suspended phases a problem associated with such a macro-micro interface is that the volume flow rates of the microfluidics, when translated into the tubing and components of the macro system is insufficient to counteract gravitational sedimentation of the suspended phase. In this system the microfluid chip results in typical flow rates of 2.5 picolitres per second. This not only has the potential to restrict the flow of cells to the microdevice, but more perniciously it can dynamically change the local density of the fluid and hence the distribution of pumping losses (pressure drops) within the system external to the microfluidics resulting in an apparent intra-experimental pressure drift.

The use of higher pressures to force higher flow rates is precluded by the lysis of any cells that are swept into, and consequently block, the channel array at elevated flow velocities. Such problems have determined a system design with direct injection of cell samples into a separate port in the lower face manifolding, furthermore a branch of the manifolding provides for priming the blood sample through to waste without invoking the precision flow structures of the upper device plane. To a very large degree such a configuration isolates the priming of the main fluid circuits and microdevice from the blood circuitry. Indeed the blood

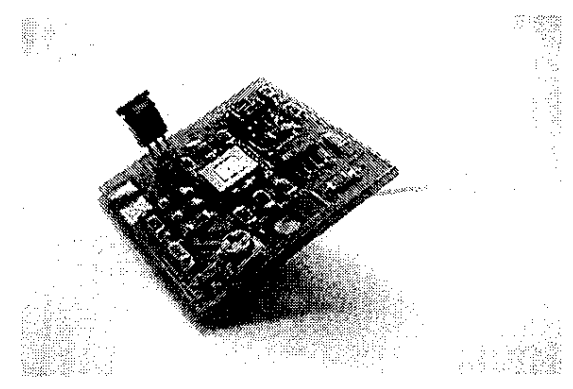

Fig. 4. A PCB-mounted, seven port microfluidic device. circuit is primed and flushed by flow directed through the manifolding away from the device.
Cells are measured at true physiological microvascular pressures in the (0-100) $\mathrm{Pa}$ range. The pressure is set by a highly precise hydrostatic head under computer control. Pressure zeroing is achieved by stopping a cell mid channel and calibrating the system at this point.

\section{Real-Time Image Processing and Data Post- processing}

Given the objective of measuring several thousand erythrocytes on a cell-by-cell basis it was never feasible to employ direct observation (although a few special purpose devices were employed in this manner). Accordingly, from the outset, the system has employed real-time, (data extraction at true video rates without frame buffering) greym scale, image processing methods to concurrently extract channel transit velocity and a volume metric for each erythrocyte in a sample of approximately 1500 .

The image processing system including the manner in which the custom-written software automatically filters out ambiguous cell transits has largely remained unchanged since this instrument was reported in [6].

One of two forms of volume metric can be obtained during analysis: a measurement of the cell length within the channel or a relative measurement of the cell volume, based on the relative absorbency by the cell of $400 \mathrm{~nm}$ incident illumination. This wavelength corresponds to an absorbency peak of haemoglobin within the cells and hence results in video images of dark cells against a reflective silicon background. A typical video image is shown in Fig. 9.

The cell volume measurements are described as metrics to recognise that they are relative measurements. This is particularly the case for measurement of cell length where a monotonic relationship between cell length and actual volume is assumed. However, the instrument's volume measurements have been shown to agree excellently with absolute volume measurements obtained using the Technicon H2 Haemocytometer: a standard haematological instrument [16].

Subsequent to the image processing stage the resultant data is analysed in simpler cases by packages such as Microsoft Excel. However for more detailed analyses we employ the technique of Kernel Density Estimation (KDE) [17]. This technique provides insight into the spatial density of cells within volume-velocity space.

\section{METHODS}

\section{A. Fluidic Operating Procedure}

Bubbles are a notorious practical problem in microfluid systems. In our system they can manifest themselves in two ways: if they lodge in narrow cross-section areas of the microfluidics they can totally interrupt fluidic operation by virtue of the high net meniscus forces they possess. Conversely, in larger bore, millifluid, areas they may not occlude the channel, but rather gently float upwards causing an associated pumping effect. In this system errors of a few Pascals are sufficient to invalidate experiments and we have observed such errors being induced by flowing bubbles.

In order to ensure a bubble-free fluid system, the

$64 \quad 1^{\text {st }}$ Annual Intemational IEEE-EMBS Special Topic Conference on Microtechnologies in Medicine \& Biology October 12-14, 2000, Lyon, France - 0-7803-6603-4/00/\$10.00@2000 IEEE 
complete fluid circuit is first primed with high purity carbon dioxide [18] and then with $1.2 \mu \mathrm{m}$ filtered, degassed phosphate buffered saline (PBS). The microfluidic device is then primed with the erythrocyte suspension by application of a pneumatic over-pressure. Having primed the cells to the lower face of the device, cell flow through the channels is controlled by application of a hydrostatic pressure differential across the device.

When multiple samples are tested within a single experiment, the fluid system is flushed with PBS between samples.

\section{B. Blood Preparation}

1) Normal blood : $3 \mathrm{ml}$ of blood was obtained from a healthy adult via venepuncture. Following centrifugation for five minutes at $3000 \mathrm{~g}$ the plasma was aspirated from the sample and the white bloods cells and platelets were carefully removed and discarded. The erythrocytes were resuspended in PBS then washed three times. Finally, the erythrocytes were suspended, at a haematocrit (cell concentration by volume) of $3 \%$, in PBS containing $5 \%$ autologous plasma. Prior to use, all suspending media were filtered to $1.2 \mu \mathrm{m}$. All samples of normal blood presented in this paper were prepared in this manner.

2) Artificially treated cells : Erythrocytes from a $3 \mathrm{ml}$ normal sample were isolated and washed as described above. The cells were then re-suspended at a $10 \%$ haematocrit in PBS containing $0.3 \mathrm{mg} / \mathrm{ml}$ phenylhydrazine hydrochloride (Sigma Chemicals) and incubated for 1 hour at $37 \mathrm{C}$. The erythrocytes were then washed once more and re-suspended in $5 \%$ autologous plasma as above.

3) Thalassaemic cells : $10 \mathrm{ml}$ blood samples were withdrawn, immediately prior to transfusion, from a splenectomised, $\beta$-thalassaemia-major patient attending the Haematology Clinic of the Royal Free Hospital, London. The sample was prepared as per normal blood.

\section{RESULTS AND DISCUSSION}

\section{A. Normal Blood}

It was discussed earlier that the ultimate data output of the instrument is a concurrent measurement of the velocity and length, or volume index, of each erythrocyte in a large sample as it transits a microchannel within the microfluidic device. The plot shown in Fig. 5 is a typical example of a sample of healthy adult erythrocytes. This data was obtained for cells passing through channels of $3.0 \mu \mathrm{m}$ width $\times 3.2 \mu \mathrm{m}$ depth $\times 100 \mu \mathrm{m}$ length, under a driving pressure of $5 \mathrm{~mm}$ of $\mathrm{H}_{2} \mathrm{O}$.

The most distinctive feature of the results shown in Fig. 5 is the highly significant $(P<0.0005, r=-0.82)$ inverse linear relationship between erythrocyte length and velocity. It should be emphasised here that each point on the scatterplot represents a paired measurement of a single cell. In this case the sample size was 1491.

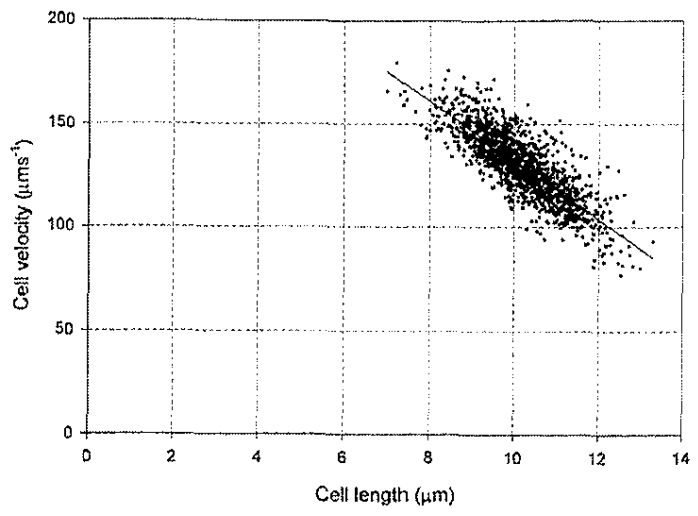

Fig. 5. Erythrocyte length/velocity scatterplot of a normal sample.

By way of illustrating the inter-experimental reproducibility of the instrument, largely in terms of the repeatability of pressure setting accuracy, multiple measurements were made on a single sample under identical operating conditions. Fig. 6 shows the linear fits of the three worst matched (for the sake of clarity) of nine experimental runs performed using the same channel array.

Whilst a thorough statistical analysis of the results is not presented here, Fig. 6 serves to indicate the high level of experimental repeatability. Clearly this is an essential prerequisite to the serious analysis of biophysical or clinical samples.

Reference [9] reports manual, paired cell size and velocity measurements of erythrocytes passing through repeated banks of $12 \mu \mathrm{m}$ long microchannels. Resting erythrocyte diameter was taken as a measure of cell size. Ensuing measurements of 23 cells yielded no significant correlation between cell size and velocity: a result in sharp contrast to those presented here. In the absence of any reported detail of the supporting fluid system, or indication of the fidelity of pressure control achieved, a loss of isobaric conditions during the course of measurements may well explain their lack of correlation.

\section{B. Chemically Treated Blood}

Identification of sub-populations of rigid cells is deemed important when evaluating pathologically altered blood [19]. To illustrate the resolution afforded by a paired, bi-

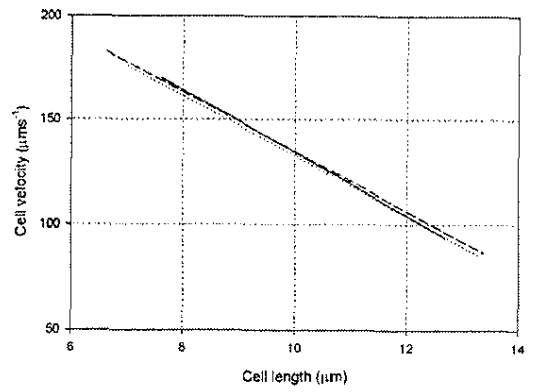

Fig. 6. Comparison of the linear regression lines for data obtained from three identical experimental runs.

variate measurement process, an erythrocyte suspension 
containing a $10 \%$ sub-population of artificially treated cells within a normal sample was analysed. The sub-population was treated with phenylhydrazine hydrochloride to reduce the intrinsic mechanical deformability of the cell membranes. Fig. 7a presents the results of such a sample, measured in a channel array containing channels measuring 3.5 wide $\mu \mathrm{m} \times 3.2 \mu \mathrm{m}$ deep $\times 100 \mu \mathrm{m}$ long. The driving pressure was $7 \mathrm{~mm}$ of $\mathrm{H}_{2} \mathrm{O}$. For comparison, a pure sample of the normal cells was also measured under identical conditions and these results are shown in Fig. $7 \mathrm{~b}$.

The existence of a sub-population of abnormally slow moving cells is clearly evident from Fig. 7a. If we consider only a uni-variate presentation of the data as shown in the figure inset which presents the velocity distribution of the sample, then the sub-population is not apparent. Whilst there appears to be a low velocity tail to the distribution, it is impossible to determine the mechanism responsible. An erythrocyte sample with an extended volume range may well produce such a velocity distribution. The concurrent measurement of velocity and a volume index allows the sub-population to be clearly differentiated from the normal population. Revealingly, it can be seen that the slow moving cells are of normal size, thus clearly indicating some impairment of the intrinsic material mechanical properties of these cells.
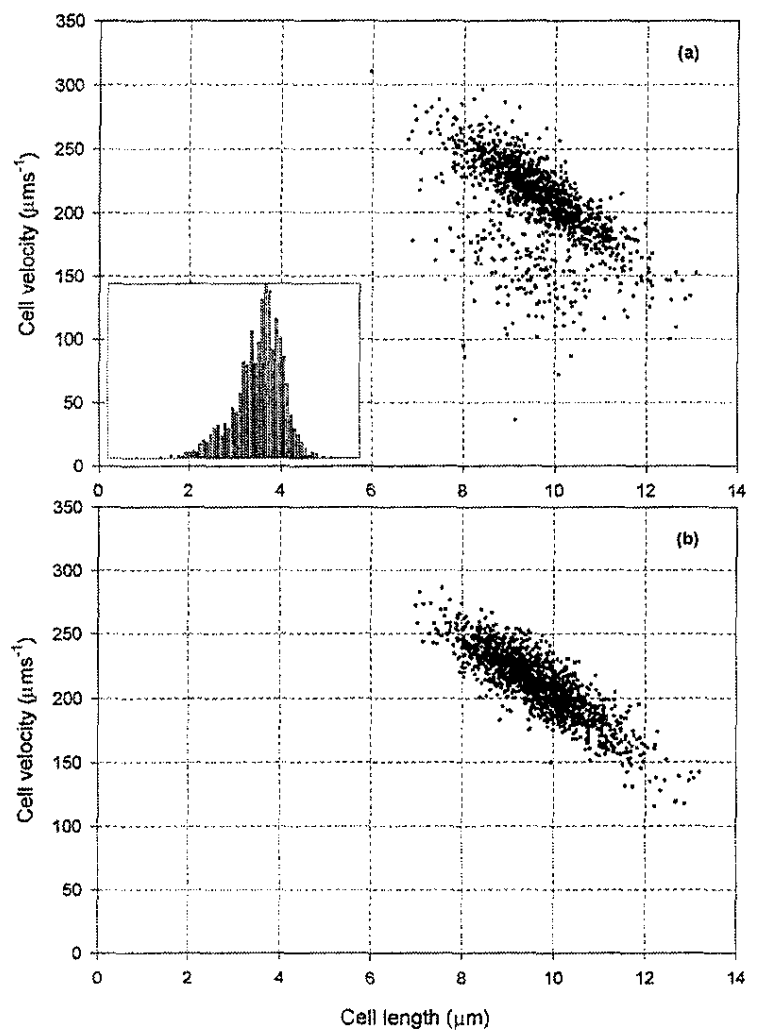

Fig. 7. a) Erythrocyte length/velocity scatterplot of a normal cell sample containing a $10 \%$ (by volume) sub-population of chemically hardened cells. The inset shows the velocity distribution of the same data. b) Erythrocyte length/velocity scatterplot of a pure sample of the normal cells.

\section{Thalassaemic Blood}

Thalassaemia is a pathological condition in which the erythrocytes are haematologically abnormal and also exhibit impaired cellular deformability [20]. Fig. 8a shows length and velocity data of erythrocytes obtained from a transfused $\beta$-thalassaemic patient. The channels used here were $3.0 \mu \mathrm{m}$ wide $\times 3.3 \mu \mathrm{m}$ deep $\times 100 \mu \mathrm{m}$ long and the driving pressure was $5 \mathrm{~mm}$ of $\mathrm{H}_{2} \mathrm{O}$. The figure inset shows a contour plot of the same data, constructed using KDE techniques. Again, for comparison, a sample of healthy adult control erythrocytes was measured under identical conditions and the results are shown in Fig. $8 \mathrm{~b}$.

Perhaps the most fundamental observation to be made about Fig. 8 is that the thalassaemic sample is profoundly abnormal by inspection. There is an obvious extension of the erythrocyte length distribution towards smaller cells. Such microcytosis is a haematological characteristic of thalassaemia and this result was indeed confirmed by conventional haematological analysis (Technicon $\mathrm{H}_{2}$ ).

From a haemorheological perspective, the salient feature of the thalassaemic data is the relatively large scatter of data points showing a number of cells having markedly higher velocities than other cells of similar size. By visual analysis of the data and the experimental videotape (each measured cell also has its time of channel entry recorded) it could be

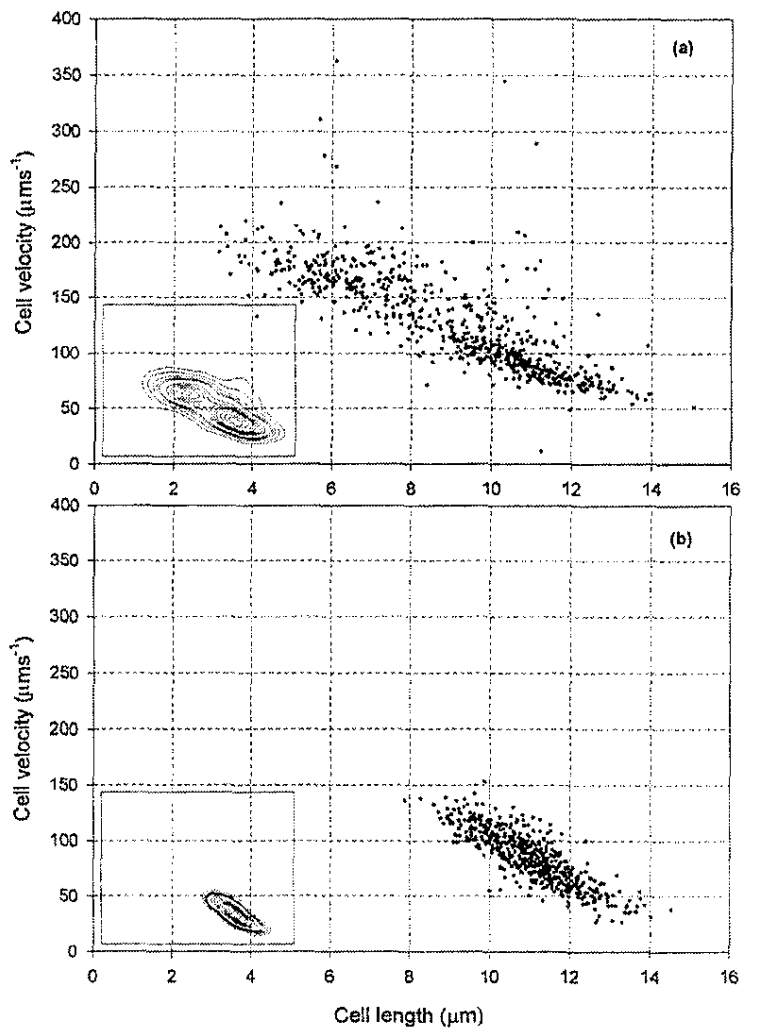

Fig. 8. a) Erythrocyte length/velocity scatterplot of cells from a transfused, splenectomised thalassaemic patient. b) Erythrocyte length/velocity scatterplot of cells from a healthy control. The figure insets show KDE contour plots of the data.

seen that these fast cells were very clearly haemoglobin- 
poor or hypochromic.

The thalassaemic patient had undergone a blood transfusion four weeks prior to blood sample collection: a fact which leads to a certain ambiguity in interpreting the results, as the sample inherently included two distinct cell populations. Looking at Fig. $8 \mathrm{a}$ and Fig. $8 \mathrm{~b}$ in conjunction, one interpretation of the data is that the transfused cells are shown as the high datapoint density region appearing towards the large end of the cell range (approximately $9 \mu \mathrm{m}$ and greater) on Fig. 8a. The microcytic and larger hypochromic cells are assumed to be thalassaemic. The contour plot representations of the data emphasise the relatively good mapping between the 'transfused' population of the thalassaemic sample and the normal sample in Fig. 8 b.

It is clear from Fig. 8 that the microchannel flow of these thalassaemic erythrocytes is not detrimentally affected when compared with normal cells. Although intrinsic mechanical properties of thalassaemic cells have been shown to be impaired [20], here we suggest that due to their microcytosis and hypochromia, thalassaemic erythrocytes may actually exhibit beneficial flow properties in a microvessel environment.

\section{CONCLUSIONS AND FURTHER WORK}

The employment of precise microfluidic components allied with a very carefully optimised fluid control and data extraction system has allowed us to investigate the microflow properties of normal, chemically altered and pathological erythrocytes to a precision hitherto impossible. In particular we have measured samples of circa 1500 cells characterising each cell in terms of a volume index and flow velocity. By this means we can independently examine the flow of a cell in terms of its intrinsic cell-membrane mechanical properties and its extrinsic property of volume.

We are currently experimenting with more advanced flow channels of varying section as shown in Fig. 9 which sequentially subject the cell to static, and then dynamic, deformation. This offers the prospect of separating effects due to changes in the elastic modulus of the cell membrane, governing static deformation, from their viscous component, governing the dynamic behaviour.

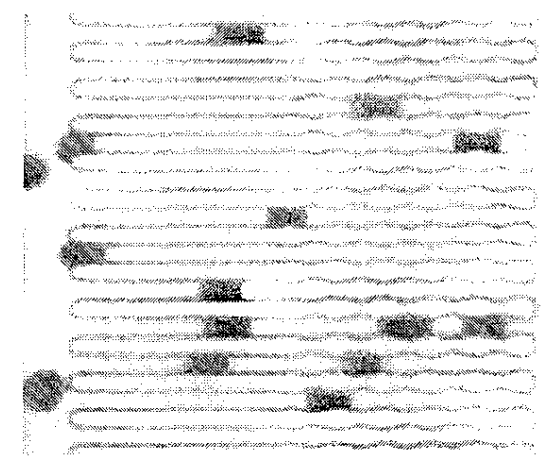

Fig. 9. Image of erythrocytes flowing through channels containing parallel-walled and varying cross-section elements for observing static and dynamic cellular deformation. Flow is from left to right. ACKNOWLEDGMENT
We acknowledge the contributions of our colleagues Guido Schulze, Carsten Kleinsteuber and Mathias Dittmann at TU-Chemnitz; Richard Greenaway and John Davis at UH; Mike Rampling at Imperial College, London and Adrian Barnes at the Wellhouse NHS Trust.

\section{REFERENCES}

[1] M. Paulitschke, and G. B. Nash, "Micropipette methods for analysing blood cell rheology and their application to clinical research," Clin. Haemorheol., vol. 13, pp. 407-434, 1993.

[2] G. B. Nash, "Filterability of blood cells: methods and clinical applications," Clin. Haemorheol, vol. 10, pp. 353-362, 1990

[3] Y. Kikuchi, H. Ohki, T. Kaneko, and K. Sato, "Microchannels made on silicon wafer for measurement of flow properties of blood cells,"Biorheology, vol. 26, p. 1055, 1989.

[4] M. C. Tracey, P. H. Kaye, and J. N. Shepherd, "Microfabricated microhaemorheometer," in Rec. 6ih Int. Conf. Sensors Actuators, California, pp. 82-84, 1991

[5] M. C. Tracey, R. S. Greenaway, A. Das, P. H. Kaye, and A. J. Barnes, "A silicon micromachined device for use in blood cell deformability studies," IEEE Trans. Biomed. Eng., vol. 42, pp. 751$761,1995$.

[6] N. Sutton, M. C. Tracey, I. D. Johnston, R. S. Greenaway, and M. W. Rampling, "A novel instrument for studying the flow behaviour of erythrocytes through microchannels simulating human blood capillaties," Microvasc. Res., vol. 53, pp. 272-281, 1997.

[7] G. R. Cokelet, R. Soave, G. Pugh, and L. Rathbun, "Fabrication of in vitro microvascular blood flow systems by photolithography." Microvasc. Res., vol. 46, pp. 394-400. 1993.

[8] P. Wilding, J. Pfahler, H. H. Bau, J. N. Zemel, and L. J. Kricka, "Manipulation and flow of biological fluids in straight channels micromachined in silicon," Clin. Chem., vol. 40, pp. 43-47, 1994.

[9] J. P. Brody, Y. Han, R. H. Austin, and M. Bitensky, "Deformation and flow of red blood cells in a synthetic lattice: evidence for an active cytoskeleton," Biophys. $J$. vol. 68 , pp. 2224-2232, 1995.

[10] P. Wilding, L. J. Kricka, J. Cheng, G. Hvichia, M. A. Shoffner, and P. Fortina, "Integrated cell isolations and polymerase chain reaction analysis using silicon microfilter chambers," Anal. Biochem, vol. 257, pp. 95-100, 1998

[11] P. Telleman, U. D. Larsen, J. Philip, G. Blankenstein, and A. Wolff, "Cell sorting in microfluidic systems," in Proc. $\mu$ TTAS '98. Banff, pp. $39-44,1998$

[12] H. C. Zeringue, K. R. King, I. K. Glasgow, S. Raty, M. B. Wheeier, and D. J. Beebe, "Zona pellucida removal of mammalian embryos in a microfluidic system," in Proc. $\mu$ TAS 2000, Twente, pp. 435-438, 2000.

[13] R. D. Olescuk, A. B. Jemere, L. L. Schultz-Lockyear, F. Fajuyigbe, and D. J. Harrison, "Utilization of bead based reagents in microtluidic systems," in Proc. $\mu T A S$ '2000. Twente, pp. 11-14, 2000 .

[14] M. Tracey, I. Johnston, R. Greenaway, J. Davis, N. Sutton, G. Schulze, and W. Doetzel, "A microfluidics-based microcytometer interfacing microfluidics with macrofluidics," in Proc. $\mu T A S 1998$, Banff, pp. 89-92, 1998

[15] A. E. Holdo, M. C. Tracey, and J. B. Davis, "The use of CFD for microhaemorheometer design," in Proc. NAFEMS World Congress 99, pp. $517-528,1999$.

[16] R. S. Greenaway, "Image processing and data analysis algorithms for application in haemocytometry," $\mathrm{PhD}$ Thesis, University of Hertfordshire, 1998

[17] B. W. Silverman, Density estimation for statistics and data analysis, Chapman and Hall, NY, 1986.

[18] R. Zengerle, M. Leitner, S. Kluge, and A. Richter, "Carbon dioxide priming of micro liquid systems," Proc. MEMS "95, Amsterdam, pp. 19-24. 1995.

[19] J. Stuart and G. B. Nash, "Red cell deformability and hacmatological disorders," Blood Rev., vol. 4, pp. 141-147, 1990.

[20] G. Athanasiou, N. Zoubos, and Y. Missirlis, "Erythrocyte membranc deformability in patients with thalassemia syndromes," Nouv. Rev. Fr. Hematol., vol. 33, pp. 15-20, 1991. 